\title{
1 Causation without correlation: parasite-mediated frequency-dependent \\ 2 selection and infection prevalence
}

6 Curtis M. Lively ${ }^{1}$, Julie $\mathrm{Xu}^{1}$, Frida Ben-Ami ${ }^{2}$

$12{ }^{2}$ School of Zoology, George S. Wise Faculty of Life Sciences, Tel Aviv University, Tel

13 Aviv 6997801, Israel

17 Author for correspondence. C.M. Lively, e-mail: clively@indiana.edu 
22 Parasite-mediated selection is thought to maintain host genetic diversity for resistance.

23 We might thus expect to find a strong positive correlation between host genetic diversity

24 and infection prevalence across natural populations. Here we used computer simulations

25 to examine host-parasite coevolution in 20 simi-isolated clonal populations across a

26 broad range of values for both parasite virulence and parasite fecundity. We found that

27 the correlation between host genetic diversity and infection prevalence can be

28 significantly positive for intermediate values of parasite virulence and fecundity. But the

29 correlation can also be weak and statistically non-significant, even when parasite-

30 mediated frequency-dependent selection is the sole force maintaining host diversity.

31 Hence correlational analyses of field populations, while useful, might underestimate the

32 role of parasites in maintaining host diversity.

33

34

35 Subject Area. Evolution

36

37 Keywords: host-parasite coevolution, genetic diversity, negative frequency-dependent

38 selection 


\section{1. Introduction}

41 An appealing idea in disease ecology is that coevolving parasites act to maintain genetic

42 diversity in natural host populations $[1,2]$. The idea assumes that parasites must mimic

43 host cell-surface molecules in order to evade the host's immune system $[2,3]$. This kind

44 of system favors parasite genotypes that can infect the most common host genotypes,

45 thereby giving an advantage to rare host genotypes [2, 4-8]. Host diversity is thus

46 expected to accumulate due to parasite-mediated, negative frequency-dependent

47 selection. Under this reasoning, it seems sensible to predict that host genetic diversity

48 would be positively correlated with disease prevalence in the wild [e.g., 9, 10].

49 On the other hand, strong parasite-mediated selection can drive oscillatory

50 dynamics in both host and parasite genotype frequencies $[2,4,11,12]$. Such dynamics

51 might reduce the association between genetic diversity and parasite prevalence, at least

52 during parts of the coevolutionary cycle. In addition, high host genetic diversity might

53 reduce disease spread, leading to a lower prevalence of infection $[13,14]$. This latter idea

54 has support from agricultural studies [15-17], as well as from natural populations [e.g.,

$5518,19,20][$ recent reviews in, 21, 22].

56 The potentially complex relationship between (1) negative frequency-dependent

57 selection, (2) genetic diversity, and (3) oscillatory dynamics suggests that the association

58 between genetic diversity and disease prevalence in natural populations might be

59 complex and/or nonintuitive. In the present paper, we used simulation studies to examine

60 when coevolving parasites maintain genetic diversity in clonal host populations, and

61 when one might expect to find a significantly positive correlation between infection

62 prevalence and host genetic diversity. We found that host-parasite coevolution can lead 
63 to the maintenance of high levels of host genetic diversity, provided the strength of

64 parasite-mediated selection is stronger than host fecundity selection. We also found

65 regions of parameter space for which the correlation between host diversity and parasite

66 prevalence was positive and statistically significant; but the region is small, and it only

67 partially overlaps with the parameter space for high genetic diversity.

68

\section{2. Simulation Model}

70 We used Excel to simulate host-parasite coevolution in 20 populations. We assumed

71 clonal host reproduction, as neutral genetic markers can be used to infer different multi-

72 locus resistance genotypes from field-collected organisms. This approach is especially

73 helpful when the genetic architecture of resistance is unknown, and it has often been used

74 by field biologists to study natural host-parasite interactions, especially in freshwater

75 snails and waterfleas [e.g., 7, 10, 23, 24]. The hosts were assumed to be haploid, where

76 resistance was determined at two different loci, with three alleles at each locus, giving

77 nine different possible genotypes. Given that the hosts were assumed to be asexual, this

78 set up is the same as that for a single locus with nine alleles. The parasites were also

79 assumed to be haploid asexuals with the same complement of genotypes. We assumed a

80 matching alleles model of infection, meaning that parasites with alleles that did not match

81 their host at both loci were killed by the host's self-nonself recognition system $[25,26]$.

82 Both the hosts and the parasites were assumed to be annuals, meaning that the host and

83 parasites have synchronized life cycles.

84 The nine host clones were initiated at different randomly selected frequencies in

85 the 20 populations. To simulate fecundity selection among clones, the maximum 
86 fecundity for uninfected hosts $\left(b_{\mathrm{u}}\right)$ was then randomly assigned to each clone separately

87 in all populations to give a mean within-population fecundity of 10 with a standard

88 deviation of either 2.0 or 1.0 . This randomization process meant that the same clonal

89 genotypes would likely have had different maximum fecundities in different populations,

90 which could, for example, be a result of resource differences among populations and/or

91 differences in mutational loads among populations. The process was meant to establish

92 the kind of fecundity differences among clones that is known for freshwater snails and

93 Daphnia [e.g., 27, 28]. As expected, fecundity selection in the absence of parasites

94 rapidly eroded clonal host diversity in the simulated populations.

95 The realized fecundity for uninfected individuals (subscript $\mathrm{u}$ ) was density-

96 dependent and equal to

$$
B_{\mathrm{u}(j, k)}=\frac{b_{\mathrm{u}(j, k)}}{1+a_{\mathrm{u}} N_{k}}
$$

98 Here $b_{\mathrm{u}(j, k)}$ is the number of offspring that would be produced by uninfected individuals of

99 the $j^{\text {th }}$ clone in the $k^{\text {th }}$ population in the absence of conspecific competitors; $a_{\mathrm{u}}$ reflects the

100 sensitivity of uninfected individuals to competition in all populations, and $N_{k}$ gives the

101 total number of hosts in the $k^{\text {th }}$ population [following 29]. In the present study, we set $a_{\mathrm{u}}$

$102=0.0001$. The carrying capacity $(K)$ of an uninfected population, assuming $b_{\mathrm{u}}=10$ and $a_{\mathrm{u}}$

$103=0.0001$, is $K=\left(b_{\mathrm{u}}-1\right) / a_{\mathrm{u}}=90,000$. The carrying capacity of partially infected

104 populations can be somewhat lower.

105 The realized fecundity for infected individuals (subscript i) was calculated as a

106 proportion of the realized fecundity of uninfected individuals:

$$
B_{\mathrm{i}(j, k)}=B_{\mathrm{u}(j, k)}(1-v)
$$


108 where $v$ (for virulence) reflects the reduction in fecundity for infected individuals relative

109 to uninfected individuals. Here $\left.v=1-b_{\mathrm{i}(j, k)}\right) b_{\mathrm{u}(j, k)}$, where $b_{\mathrm{i}(j, k)}$ is the maximum number of

110 offspring produced by infected individuals of the $j^{\text {th }}$ clone in the $k^{\text {th }}$ population. In the

111 simulations, we altered virulence by changing the value for $b_{\mathrm{i}}$ relative to $b_{\mathrm{u}}$. Lower

112 values of $b_{\mathrm{i}}$ reflected higher virulence.

113 The host populations were all initiated at 90,000 total individuals. To initiate

114 disease spread, an infected adult from each host clone was introduced to all populations

115 at generation 1. In subsequent generations, hosts became infected as juveniles if they

116 contacted one or more genetically matched propagules (spores or eggs) that were shed

117 into the environment by infected hosts in the previous generation. The number of

118 infected juveniles for each host genotype in each population was calculated as,

$$
I_{j, k(t+1)}=h_{j, k(t+1)} N_{k(t+1)}\left[1-\exp \left(-\beta I_{j, k(t)} / N_{k(t+1)}\right)\right]
$$

120 where $\beta$ is the realized parasite fecundity, which gives the number of propagules

121 produced by a single infection that make contact with a host in the next time step. Note

122 that $\beta$ is expected to be a small fraction of the total number of propagules released by

123 infected hosts. $I_{j, k(t)}$ is the number of infected individuals for the $j^{\text {th }}$ host genotype in

124 population $k$ at time $t ; h_{j, k(t+1)}$ is the frequency of hosts with genotype $j$ in population $k$ at

125 time $t+1$, and $N_{k(t+1)}$ is the total number of hosts in the $k^{\text {th }}$ subpopulation. The expression

126 in square brackets gives the probability of infection, assuming that exposure to a single

127 matching parasite genotype is sufficient to cause infection. Infected hosts did not recover

128 from infection.

129 Similarly, the number of uninfected juveniles for each host genotype $j$ in each

130 population $k$ was calculated as, 


$$
U_{j, k(t+1)}=h_{j, k(t+1)} N_{k(t+1)}\left[\exp \left(-\beta I_{j, k(t)} / N_{k(t+1)}\right)\right]
$$

132 Here the term in square brackets gives the Poisson-distributed probability for the "zero

133 class," meaning that the host is not exposed to a matching parasite genotype and remains

134 uninfected. This basic approach to modeling infection dynamics was borrowed from

135 previous studies [30,31]. A recent exploration of the approach is given by [32].

136 To simulate host and parasite migration, we coded the simulation to introduce a

137 single infected individual from each host clone with a probability of 0.02 in all

138 populations. Hence, a single infected migrant for each clone was introduced, on average,

139 every 50 generations. Similarly, a single uninfected migrant for each host clone was

140 introduce with a probability of 0.05 (giving an average of one migrant every 20

141 generations). The migrants were assumed to be drawn from a very large metapopulation,

142 of which the 20 simulated populations were only a small part. The main purpose was to

143 introduce genetic variation into the parasite population. In the absence of migration,

144 parasite genetic diversity could be stochastically lost. This is especially true for highly

145 virulent parasites, which tend to generate high-amplitude oscillations that push allele

146 frequencies near to fixation during parts of the cycle [33] (Fig. 2). A total loss of parasite

147 alleles would further decouple the correlation between infection prevalence and host

148 genetic diversity. As such, the incorporation of a low level of migration to restore

149 diversity is conservative with respect to the findings reported here.

150 The simulation was run for 2100 generations, and the data from each of the 20

151 populations were collected at the final generation. This was meant to capture the system

152 at a single time point in the way in which field biologists might sample a 
153 metapopulations. At generation 2100, we calculated the prevalence of infection for each

154 population. We also calculated host genotypic diversity within each population as

$$
D_{H}=\frac{1}{n \sum h_{j}^{2}}
$$

156 where $n$ gives the number of possible genotypes in the population (here $n=9$ ), and $h_{j}$

157 gives the frequency of the $j^{\text {th }}$ host clone. This measure gives the standardized inverse

158 Simpson's index, with a maximum value of 1.0 and a minimum value of $1 / n$ [34]. We

159 also calculated the mean prevalence and mean $D_{\mathrm{H}}$ across the 20 subpopulations, as well

160 as the correlation between infection prevalence and $D_{\mathrm{H}}$ across the subpopulations. We

161 iterated the simulation for a range of values for parasite fecundity $(\beta=2,4,6,8,9,10$,

$16212,15,18,21,24,27,30,33,36)$ across a range of virulence values $(v)$ from 0.10 to 0.90

163 in increments of 0.10 . Using a standard deviation for average host fecundity equal to 2.0,

164 the 2100-generation simulation was run 1000 different times for all combinations of

165 values. The data presented are the averages of these 1000 runs for the final generation

166 (generation 2100) (Fig. 1). We repeated the whole process after reducing the standard

167 deviation for host fecundity from 2.0 to 1.0 , which reduced the effect of fecundity

168 selection. To reduce the processing time, we also reduced the number of iterations of the

169 2100-generation simulation from 1000 to 100.

\section{3. Results and Discussion}

172 Our main goal was to determine the effects of parasite fecundity and parasite virulence on

173 host genetic diversity and infection prevalence. Not surprisingly, the mean prevalence of

174 infection increased with parasite fecundity, especially when parasite virulence was low

175 (Fig. 1A,D). However, for higher levels of parasite virulence, the prevalence of infection 
176 decreased (Fig. 1A,D). This decrease in prevalence with higher levels of virulence does

177 not stem from the damping of infection, such as that observed in virulent contagious

178 diseases [e.g., 35, 36]. In our simulation models, the infection is not contagiously

179 transferred among hosts, but is rather randomly distributed among juvenile hosts in the

180 next generation. The reduction in disease is because higher levels of host genetic

181 diversity were maintained with higher levels of parasite virulence (virulence $=0.4-0.8$ ).

182 This result can be seen by comparing Figs. 1A and 1B: the transition from high to low

183 prevalence (red to blue) in Fig. 1A occurs where genetic diversity is increasing (blue to

184 red in Fig 1B).

185 Why does host-genetic diversity increase for virulence levels between roughly 0.4

186 and 0.8 ? The simplest explanation is that parasite-mediated selection becomes strong

187 enough in this region to counter the effects of fecundity selection. Given that the nine

188 host clones were initiated with variable intrinsic birth rates, the most fecund clone

189 eliminates all other clones in the absence of parasites (clonal selection). Hence, for

190 parasites having low virulence, host genetic diversity is eroded by clonal selection,

191 leading to higher levels of infection in the genetically depauperate host population. As

192 virulence increases, parasite-mediated frequency-dependent selection leads to higher

193 levels of host genetic diversity, because selection against the more common and more

194 fecund host clones counters any fecundity advantage that they have. Hence, more

195 virulent diseases lead to higher levels of host genetic diversity, which reduces the overall

196 prevalence of infection. Also note that the most common clones are most fecund when

197 uninfected, but also the most infected. This pattern would look like a trade-off in which

198 fecundity trades off with resistance. But there is no inherent trade-off. The most 
199 common local clones would not be more infected by allopatric parasites, which are

200 adapted to a different set of host genotypes.

201 Curiously, however, both genetic diversity and prevalence of infection decline as

202 the parasites become very virulent $(v>0.8)$. This leads to the hump-shaped topography

203 observed for the effects of virulence and parasite fecundity on host diversity (Fig. 1B,E).

204 To understand the pattern, we ran a "transect" through the hump. Specifically, we held

205 realized parasite fecundity constant at 15 , which meant that each infection produces 15

206 propagules that contact a host (even though 15 is likely to be a very small fraction of the

207 total propagules produced). We then evaluated the effects of different levels of virulence

208 on genotype frequency dynamics using the same simulation model as described above.

209 To do this, we calculated the mean CV (coefficient of variance) for clonal frequencies for

210 the last 100 generations of the simulation. We then took the average of these values over

211 all 20 populations. High average values would be indicative of high amplitude

212 oscillations in genotype frequencies over time.

213 The "transect" through the surface reflects the hump shaped pattern for genetic

214 diversity (plotted against virulence) shown in Fig. 2. It also shows that the genotypic

215 oscillations increased with virulence for values of virulence greater than 0.6. In addition,

216 mean diversity decreased with increasing amplitude in genotype-frequency oscillations

217 (Fig. 2), which was a direct result of different genotypes becoming periodically very

218 common over time. Thus, even though negative frequency-dependent selection is

219 maintaining variation in each of the host populations over time, the diversity in the host

220 population can periodically be relatively low. 
221 Finally, we examined the correlation between prevalence of infection and genetic

222 diversity (Fig 1 C,F). Our goal here was to determine the likelihood of detecting patterns

223 in nature when, in fact, parasites are a primary factor in maintaining host genetic

224 diversity. The results suggest that positive and significant correlations can be observed in

225 some parts of the parameter space, but this region of parameter space only partially

226 overlaps with the region of highest host genetic diversity (compare Figs 1B and 1C).

227 Hence, our results suggest that the lack of significance, or even slightly negative

228 correlations between infection prevalence and genetic diversity, cannot be taken as

229 evidence that parasites are unimportant to the maintenance of diversity in host

230 populations. Perhaps analogously, the lack of significant correlations between the

231 frequency of sexual females and the prevalence of infection in mixed (sexual and

232 asexual) populations of hosts may not be a reliable signal that parasites are unimportant

233 in selection for cross-fertilization.

\section{4. Summary}

236 We conducted a simulation study of host-parasite coevolution in a metapopulation of

237 clonal hosts to better understand the relationship between host genetic diversity and

238 prevalence of infection. In our model, the only force maintaining genetic diversity was

239 parasite-mediated frequency-dependent selection against common host genotypes.

240 Nonetheless, even in regions of parameter space showing high genetic diversity, the

241 correlation between prevalence of infection and genetic diversity could be so weak as to

242 be unlikely to be detected as statistically significant. Hence, over much of the parameter

243 space, causation does not produce significant correlation. These results suggest that field 
244 studies could lead to the false conclusion that parasites are unimportant in selecting for

245 rare genotypes, when in fact parasite-mediated selection is the only selective force

246 leading to diversity.

247

248 Data accessibility. The simulation and representative data are available from Dryad

250

251 Authors' Contributions. CML and FB-A conceived the project. CML wrote the basic

252 simulation, and FB-A wrote the excel macro. JX ran the simulations, organized the data,

253 and constructed the figures. CML and JX wrote the first draft of the paper, and FB-A and

254 CML revised the paper.

255

256 Competing Interests. We declare that we have no competing interests

258 Funding. The project was supported by grant \#2011011 from the United States-Israel

259 Binational Science Foundation (to FB-A and CML). Julie Xu was partially supported by

260 the undergraduate STARRS program at Indiana University. The final stages of the

261 project were supported by US National Science Foundation DEB-1906465 (to CML)

263 Acknowledgements. We thank Mandy Gibson, Zoe Dinges, and three anonymous

264 reviewers whose constructive suggestions helped us to improve the presentation. 


\section{References}

[1] Haldane, J.B.S. 1949 Disease and evolution. La Ricerca Scientifica (Suppl.) 19, 6876.

[2] Hamilton, W.D. 1980 Sex versus non-sex versus parasite. Oikos 35, 282-290.

[3] Burnet, F.M. 1971 "Self-recognition" in colonial marine forms and flowering plants in relation to the evolution of immunity. Nature 232, 230235.

[4] Bell, G. 1982 The masterpiece of nature: the evolution and genetics of sexuality. Berkeley, University of California Press.

[5] Jaenike, J. 1978 A hypothesis to account for the maintenance of sex within populations. Evol. Theory 3, 191-194.

[6] Wolinska, J. \& Spaak, P. 2009 The cost of being common: evidence from natural Daphnia populations. Evolution 63, 1893-1901. (doi:doi:10.1111/j.15585646.2009.00663.x).

[7] Jokela, J., Dybdahl, M.F. \& Lively, C.M. 2009 The maintenance of sex, clonal dynamics, and host-parasite coevolution in a mixed population of sexual and asexual snails. Am. Nat. 174, S43-S53.

[8] Duncan, A. \& Little, T.J. 2007 Parasite-driven genetic change in a natural population of Daphnia. Evolution 64, 796-803.

[9] King, K.C., Jokela, J. \& Lively, C.M. 2011 Parasites, sex, and clonal diversity in natural snail populations. Evolution 65, 1474-1481. (doi:10.1111/j.15585646.2010.01215.x).

[10] Dagan, Y., Liljeroos, K., Jokela, J. \& Ben-Ami, F. 2013 Clonal diversity driven by parasitism in a freshwater snail. J. Evol. Biol. 26, 2509-2519. (doi:doi: 10.1111/jeb.12245).

[11] Gandon, S. 2002 Local adaptation and the geometry of host-parasite coevolution. Ecol. Lett. 5, 246-256.

[12] Gandon, S. \& Michalakis, Y. 2002 Local adaptation, evolutionary potential and host-parasite coevolution: interactions between migration, mutation, population size and generation time. J. Evol. Biol. 15, 451-462.

[13] Lively, C.M. 2010 The effect of host genetic diversity on disease spread. Am. Nat. 175, E149-E152.

[14] King, K.C. \& Lively, C.M. 2012 Does genetic diversity limit disease spread in natural host populations? Heredity 109, 199-203. 
300 [15] Zhu, Y., Chen, H., Fan, J., Wang, Y., Li, Y., Chen, J., Yang, S., Hu, L., Leung, H.,

301 Mew, T.W., et al. 2000 Genetic diversity and disease control in rice. Nature 406, 718-

302722.

303 [16] Mundt, C.C. 2002 Use of multiline cultivars and cultivar mixtures for disease

304 management. Ann. Rev. Phytopathol. 40, 381-410.

305 (doi:10.1146/annurev.phyto.40.011402.113723).

306 [17] Mikaberidze, A., McDonald, B.A. \& Bonhoeffer, S. 2015 Developing smarter host 307 mixtures to control plant disease. Plant Pathology 64, 996-1004.

308 [18] Spielman, D., Brook, B.W., Briscoe, D.A. \& Frankham, R. 2004 Does inbreeding 309 and loss of genetic diversity decrease disease resistance? Conserv Genet 5, 439-448.

310 (doi:DOI 10.1023/B:COGE.0000041030.76598.cd).

311 [19] Altermatt, F. \& Ebert, D. 2008 Genetic diversity of Daphnia magna populations 312 enhances resistance to parasites. Ecol. Lett. 11, 918-928. (doi:10.1111/j.1461-

313 0248.2008.01203.x).

314 [20] Baer, B. \& Schmid-Hempel, P. 1999 Experimental variation in polyandry affects 315 parasite loads and fitness in a bumble-bee. Nature 397, 161-164.

316 [21] Ekroth, A., Rafaluk-Mohr, C. \& King, K.C. 2019 Host genetic diversity limits 317 parasite success beyond agricultural systems: a meta-analysis. Proc. R. Soc. B 286, 318 20191811. (doi:http://dx.doi.org/10.1098/rspb.2019.1811).

319 [22] Gibson, A.K. \& Nguyen, A.E. 2021 Does genetic diversity protect host populations 320 from parasites? A meta-analysis across natural and agricultural systems. Evolution 321 Letters 5-1, 16-32.

322 [23] Turko, P., Tellenbach, C., Keller, E., Tardent, N., Keller, B., Spaak, P. \& Wolinska, 323 J. 2018 Parasites driving host diversity: Incidence of disease correlated with Daphnia 324 clonal turnover. Evolution 72, 619-629. (doi: https://doi.org/10.1111/evo.13413).

325 [24] Duncan, A.B. \& Little, T.J. 2007 Duncan, A. B., and T. J. Little. 2007. Parasite326 driven genetic change in a natural population of Daphnia. Evolution 61, 796-803.

327 [25] Luijckx, P., Fienberg, H., Duneau, D. \& Ebert, D. 2013 A matching-allele model 328 explains host resistance to parasites. Curr Biol 23, 1085-1088.

329 (doi:10.1016/j.cub.2013.04.064).

330 [26] Agrawal, A.F. \& Lively, C.M. 2002 Infection genetics: gene-for-gene versus 331 matching-allele models, and all points in between. Evol. Ecol. Res. 4, 79-90.

332 [27] Paczesniak, D., Klappert, K., Kopp, K., Neiman, M., Seppälä, K., Lively, C.M. \& 333 Jokela, J. 2019 Parasite resistance predicts fitness better than fecundity in a natural 334 population of the freshwater snail Potamopyrgus antipodarum. Evolution 73, 1634-1646. 
335 [28] Hall, S.R., Becker, C.R., Duffy, M.A. \& Cáceres, C.E. 2010 Variation in resource 336 acquisition and use among host clones creates key epidemiological trade - offs. The

337 American Naturalist 176, 557-565.

338 [29] Maynard Smith, J. \& Slatkin, M. 1973 Stability of predator-prey systems. Ecology 339 54, 384-391.

340 [30] Lively, C.M. 2018 Habitat heterogeneity, host population structure, and parasite 341 local adaptation. Journal of Heredity 109, 29-37.

342 [31] Lively, C.M. 2010 An epidemiological model of host-parasite coevolution and sex. 343 J. Evol. Biol. 23, 1490-1497.

344 [32] Wallace, R., Liebman, A., Bergmann, L. \& Wallace, R. 2020 Agribusiness vs. public 345 health: disease control in resource-asymmetric conflict. hal-02513883.

346 [33] Lively, C.M. 1999 Migration, virulence, and the geographic mosaic of adaptation by 347 parasites. Am. Nat. 153, S34-S47.

348 [34] Nei, M. 1987 Molecular evolutionary genetics. New York, NY, Columbia 349 University Press.

350 [35] Bedhomme, S., Agnew, P., Vital, Y., Sidobre, C. \& Michalakis, Y. 2005 Prevalence351 dependent costs of parasite virulence. Plos Biol. 3, 1403-1408.

352 [36] Myers, J.H. \& Rothman, L.E. 1995 Virulence \& transmission of infectious diseases 353 in humans and insects. Trends Ecol. Evol. 10, 194-198.

354 [37] Lively, C.M., Ben-Ami, F. \& Xu, J. Data from: Causation without correlation: 355 parasite-mediated frequency-dependent selection and infection prevalence. Dryad Digital 356 Repository. (doi:https://10.5061/dryad.w9ghx3fqp). 


\section{Figure headings.}

360 Figure 1. Simulation results. The left-hand column (A-C) gives simulation results for

361 which the standard deviation in host fecundity was 2.0, while the right-hand column (D-

362 F) gives results for a standard deviation in host fecundity of 1.0. The higher standard

363 deviation (A-C) gives stronger fecundity selection. The top row (A \& D) gives the

364 results for mean infection. The middle row (B \& E) gives the results for mean diversity,

365 while the lowest row (C \& E) gives the correlation between diversity and prevalence of

366 infection. The side bars show the values of the different colors for prevalence (A \& D),

367 diversity (B \& E), and the correlation between diversity and prevalence. For graphs C

368 and $\mathrm{E}$, correlations having an absolute value of greater than 0.45 have $P$ values less than

3690.05 . Note that, as expected, high levels of diversity are maintained across more of the

370 parameter space when fecundity selection is weaker. Note also that high correlations are

371 shifted to the South and West relative to regions of high host diversity. As such, high

372 levels of host diversity can be associated with low (or even negative) correlations

373 between diversity and prevalence. Note that the simulation results assumed clonal

374 reproduction, but they could also be applicable to highly inbred populations.

376 Figure 2. Cross section through Figs $1 \mathrm{~B} \& \mathrm{C}$ at parasite fecundity $=15$. The lines

377 show the relationships between host genetic diversity (red line), fluctuations in diversity

378 (blue line, the coefficient of variation for host diversity), and the correlation between host

379 genetic diversity and infection prevalence (dashed grey line). Note that the correlation is

380 only positive and high for a small portion of parameter space in which diversity is high.

381 Also note that the peak correlation corresponds to moderate fluctuations in diversity over 
382 time (grey line). Insert, upper left: representative host genotype dynamics for moderate

383 virulence $(v=0.4)$. The different colored lines represent different host genotypes. Insert,

384 upper right: representative host genotype dynamics for moderately high virulence $(v=$

385 0.7). Insert, lower right: representative host genotype dynamics for high virulence $(v=$

386 0.8). Taken together, the results suggest that detection of strong positive correlations

387 would be most likely at moderate-to-high levels of parasite-mediated selection,

388 depending on parasite fecundity (Fig. 1). 
STD $=2$

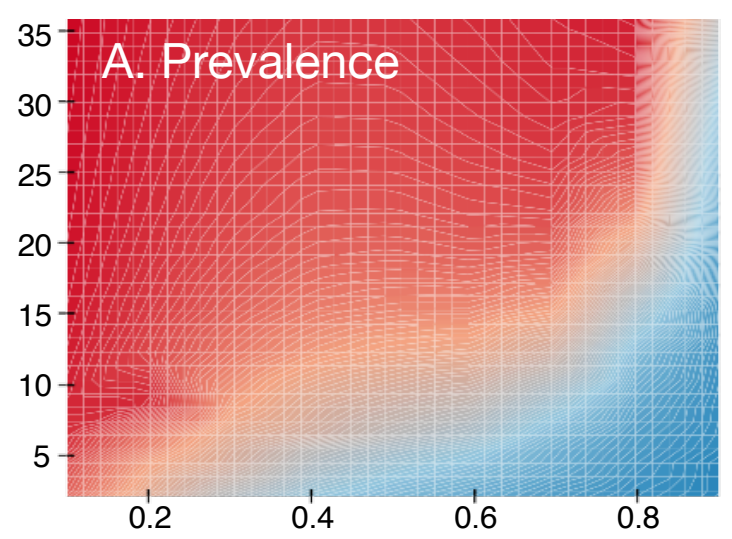

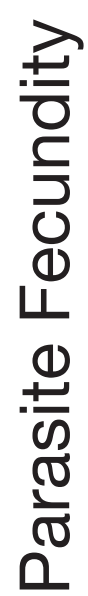
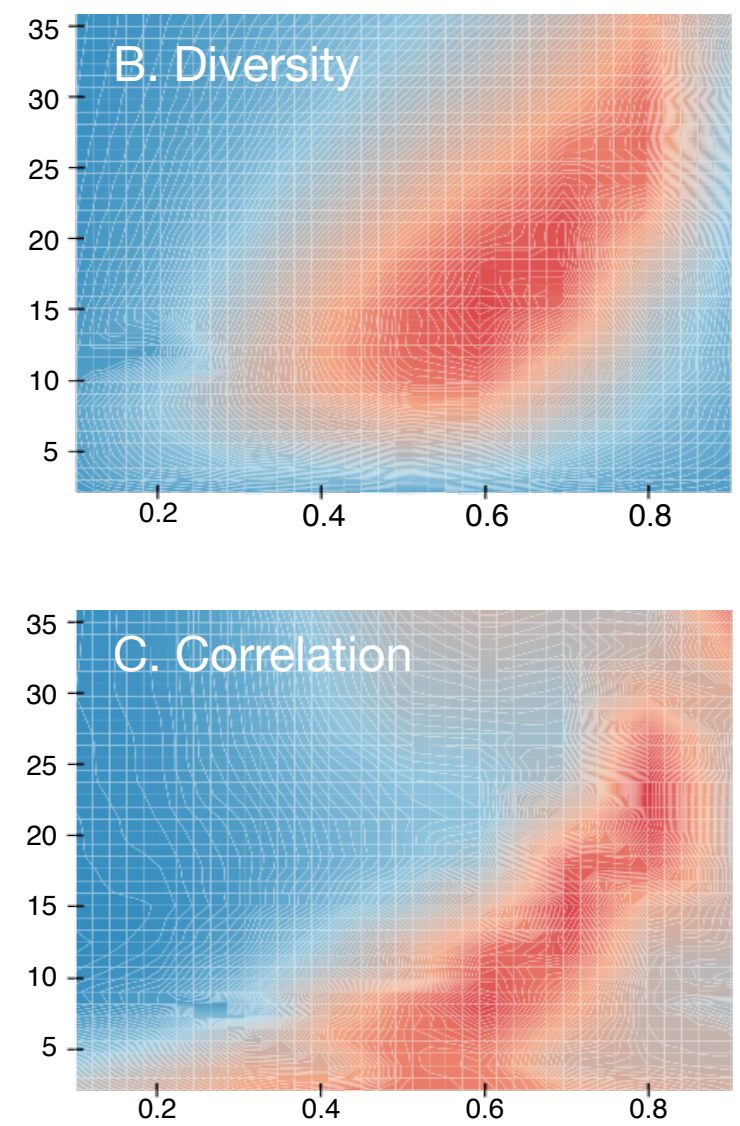

STD $=1$
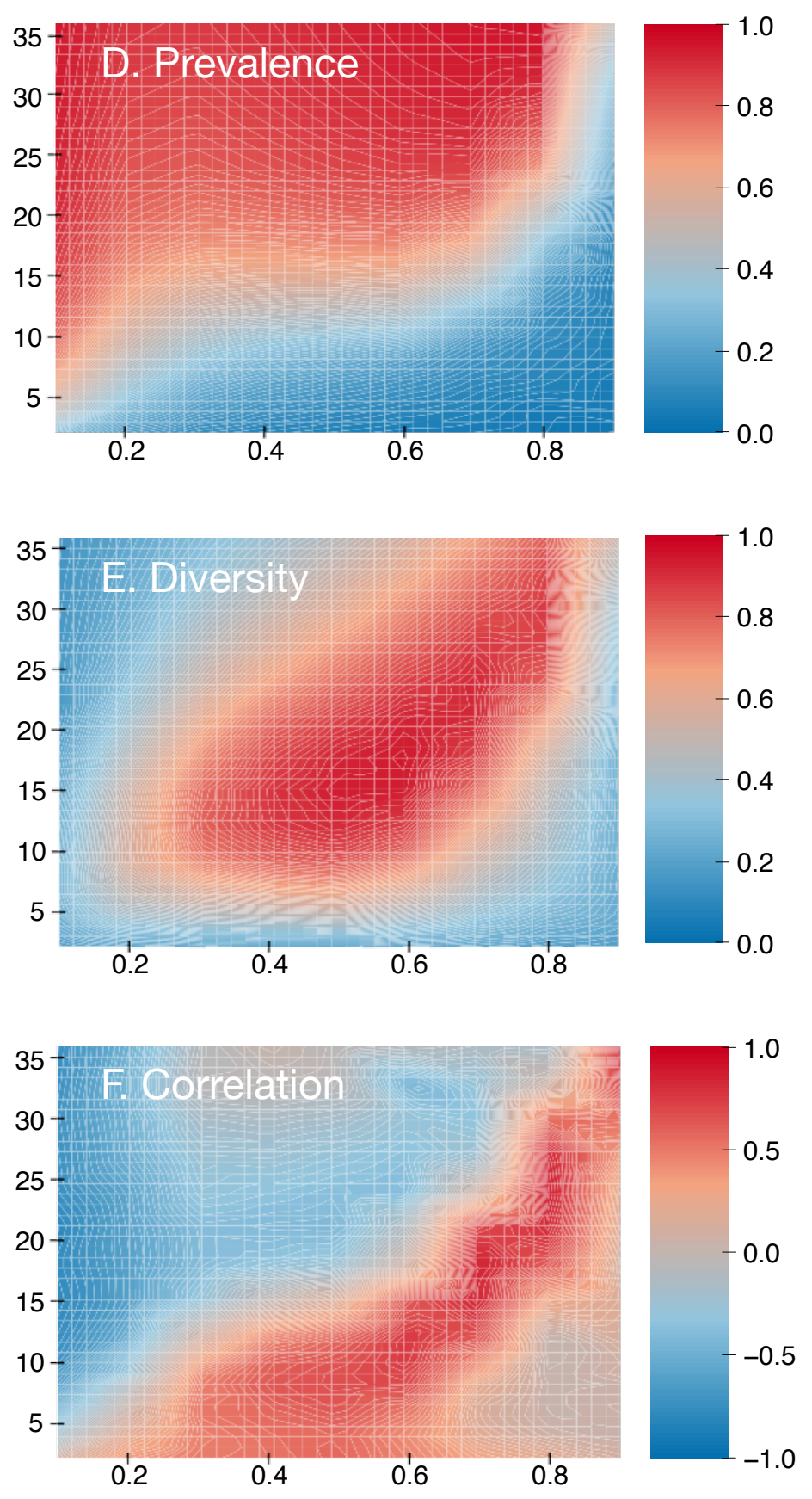

\section{Parasite Virulence}




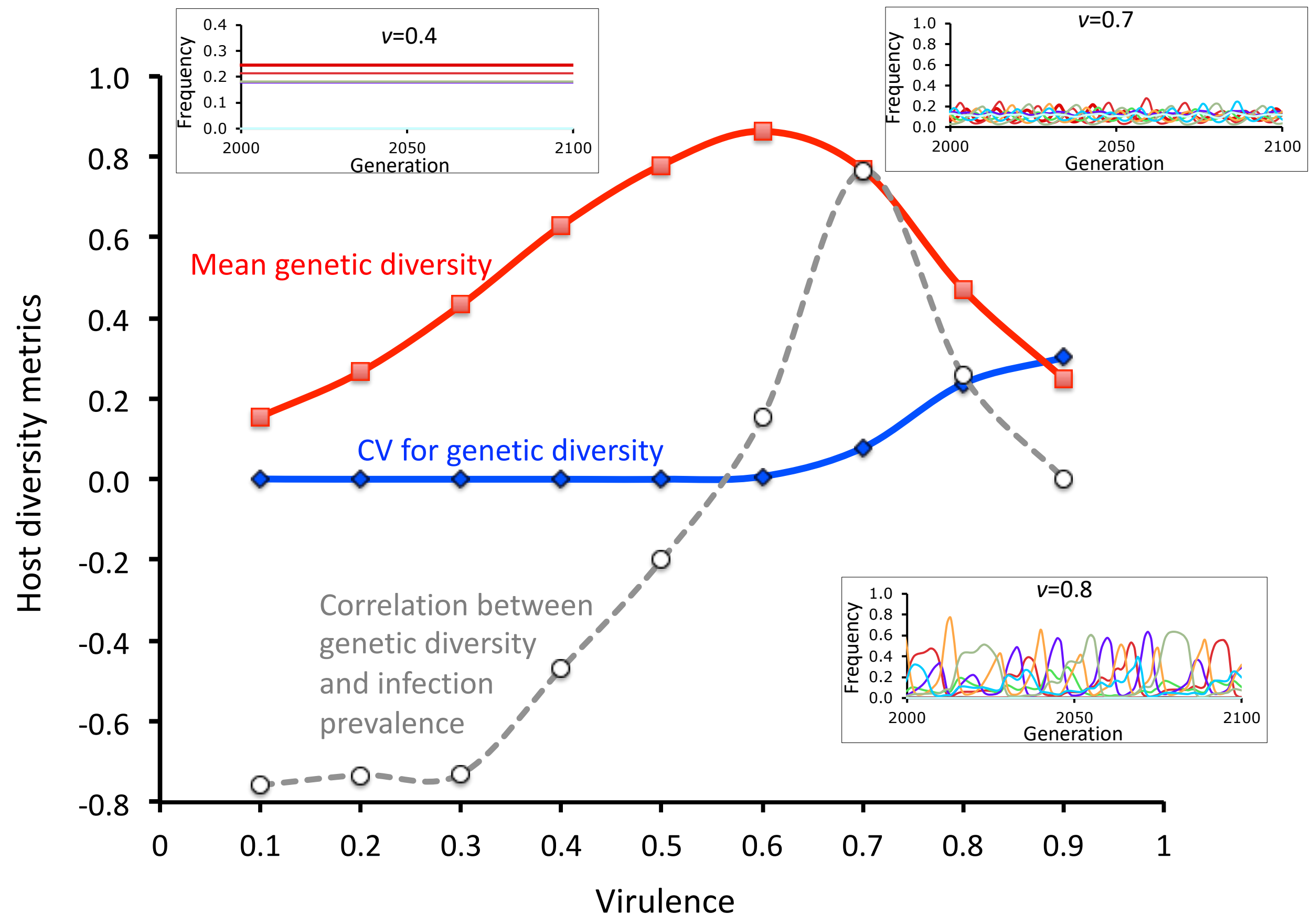

